\title{
Réception des relations de voyage en Nouvelle-France dans quelques histoires littéraires du Québec et du Canada
}

\author{
Sébastien Côté \\ Université Carleton
}

Pour ce numéro thématique consacré aux genres littéraires, j'ai choisi de poursuivre une exploration entamée il y a quelques années déjà, c'est-à-dire un débroussaillage de la tradition historiographique québécoise, destiné à mettre en évidence son attitude à l'égard du patrimoine lettré de la Nouvelle-France. Toutefois, dans le présent article, au lieu d'adopter une perspective panoramique et généraliste, je me concentrerai plus modestement sur la réception des relations de voyage dans une sélection d'ouvrages d'histoire de la littérature canadienne, canadienne-française ou québécoise publiés depuis la fin du 
19e siècle. Peu prisée par la critique historienne, souvent nationaliste, à cause de sa nature forcément exogène, en apparence informe, et, surtout, dénuée de prestige, la relation de voyage fait depuis longtemps l'objet de jugements négatifs, quand elle n'est pas tout simplement ignorée. Il est vrai que, comme le souligne Réal Ouellet, « [p]ar rapport à l'épopée et au roman, la relation de voyage occupe la même position que l'histoire ou la géographie : elle n'a pas pour objectif déclaré le fictionnel et le littéraire » (p. 1). En dépit de ces résistances, la relation de voyage occupe une place bien à elle dans le discours historiographique. Ainsi, au moyen d'une mise en contexte étayée de quelques exemples tirés d'histoires littéraires publiées au Québec depuis la fin du 19e siècle, j'esquisserai un portrait d'ensemble de la réception des œuvres appartenant à ce « genre protéiforme » (ibid.) et mal aimé. Puisque l'étude de ce vaste corpus dépasse largement les objectifs d'un article de synthèse, je me suis concentré sur trois titres en particulier : $A$ History of Canadian Literature (1989) de William H. New; Kanadische Literaturgeschichte (2005), sous la direction de Konrad Groß, Wolfgang Klooß et Reingard M. Nischik ; et, enfin, Anthologie. Confrontation des écrivains d'hier à aujourd'hui. Tome 3 : de la Nouvelle-France au Québec actuel (2007), sous la direction d'André G. Turcotte. S'il apparaît éclectique de prime abord, ce choix se justifie aisément, comme je le montrerai plus loin. En effet, ces titres présentent trois perspectives distinctes sur le corpus étudié: pancanadienne, germanophone et québécoise (voire cégépienne).

Passer en revue, pinceau de l'archéologue à la main, les strates d'un métadiscours abordant le patrimoine lettré de la Nouvelle-France, ici plus particulièrement le discours sur la relation de voyage, voilà un exercice qui peut sembler des plus 
rébarbatifs. Pourquoi, en effet, examiner de tels artéfacts au moyen d'une coupe transversale, sinon pour mettre au jour une espèce d'ordonnancement axiologique et forcément daté des goûts littéraires ? C'est justement à cette tâche que s'est attelée Karine Cellard dans Leçons de littérature: Un siècle de manuels scolaires au Québec. Elle y montre tout l'intérêt que présente un examen minutieux d'ouvrages qui, le plus souvent, ont des visées pédagogiques parfaitement assumées. Dans l'introduction, elle écrit avec justesse :

C'est que la littérature, comme toutes les sciences humaines et disciplines artistiques, n'est pas une matière neutre et son enseignement ne peut se limiter à la transmission d'un contenu objectif. Lorsqu'il s'agit de présenter une tradition littéraire qui plus est la tradition nationale - , un ensemble complexe de facteurs est susceptible de se mêler aux strictes considérations disciplinaires pour en infléchir le discours (p. 10).

Plus loin, elle applique ce principe général à l'étude de la tradition québécoise :

Il va sans dire que la mythologie de la littérature québécoise s'est complètement transformée entre la publication du premier Manuel d'histoire de la littérature canadienne-française proposée par Mgr Camille Roy en 1918 et les synthèses proRévolution tranquille publiées en 1996 pour répondre aux exigences de la réforme Robillard. C'est à ce travail progressif sur la tradition que donne accès une telle étude panoramique, attentive tant au remaniement du canon littéraire qu'aux glissements dans la conception de l'identité nationale ou au rapport même à l'œuvre lue. (p. 12)

Bref, la manière dont une institution donnée prend la parole pour façonner à rebours une tradition littéraire, dans le but notamment de la transmettre par l'entremise de l'école, peut épouser diverses formes. 
Pour accéder au statut de classique, je le rappelle, il faut qu'une œuvre ainsi désignée s'inscrive dans une histoire littéraire particulière où se serait opérée, comme l'écrit Alain Vaillant, « la formation progressive d'une tradition historique et d'un panthéon des grands auteurs, dignes d'être imités » (p. 22). Cette tradition historique, dont l'idée se cristallise au cours du 19e siècle européen (du moins, en ce qui concerne l'espace culturel occidental), a presque forcément un cadre national. C'est d'ailleurs en ces termes que Gustave Lanson conclut «La méthode de l'histoire littéraire», son célèbre article de 1910 :

La critique, dogmatique, fantaisiste, ou passionnée, divise: l'histoire littéraire réunit, comme la science dont l'esprit l'inspire. Elle devient ainsi un moyen de rapprochement entre des compatriotes que tout le reste sépare et oppose, et c'est pourquoi j'oserais dire que nous ne travaillons pas seulement pour la vérité ni pour l'humanité : nous travaillons pour la patrie. (p. 56)

Or, selon Cellard, c'est précisément ce qu'ont fait la plupart des auteurs de manuels d'histoire littéraire du Canada français tout au long du 20e siècle, mais dans le cadre particulier d'une littérature perçue comme mineure par rapport à la tradition française. Ils y sont notamment parvenus au moyen d'astucieuses "mises en intrigue »: "Comme toute entreprise historiographique, l'histoire littéraire élabore un récit qui, sous les dehors rassurants de l'objectivité, propose l'interprétation personnelle d'un corpus donné à un certain moment du temps » (p. 15). C'est à un examen de ce genre, quoique plus spécifique, que je voudrais consacrer les pages qui suivent. Toutefois, avant d'entrer dans le vif du sujet, il convient de rappeler sommairement le contexte de réception québécoise des écrits de la Nouvelle-France, en particulier des relations de voyage. 
En se penchant sur les développements de l'écriture de l'histoire littéraire du Québec depuis la fin du 19e siècle, on pourrait s'étonner du traitement réservé au corpus du Régime français, a fortiori de l'appréciation de la relation de voyage. À ce sujet, le point de vue d'Yvan Lamonde, qui a envisagé certaines de ces questions dans la perspective de l'histoire du livre et des institutions, me semble éclairant. En effet, dans Histoire sociale des idées au Québec (1760-1896), il résume en ces termes le processus général de construction identitaire :

Durant la seconde moitié du XIXe siècle, en raison du développement du Bas-Canada, du Canada-Uni et du Canada, les «métropoles » politico-culturelles du Canada français se font plus présentes dans la recherche de son identité. La référence aux héritages culturels et politiques extérieurs du Canada fiançais se fait plus fréquente et oblige à prendre la mesure du poids relatif de la France, de l'Angleterre, des ÉtatsUnis et de Rome dans l'identité en construction du Canada français. (p. 383) ${ }^{1}$

Bien que manifeste, l'héritage français se mêle désormais aux apports subséquents, ce qui se reflète également dans le choix des premières œuvres canoniques. Ainsi, au moment où, en Europe, des pays comme la France et l'Allemagne puisaient à même leur tradition linguistique séculaire afin de se constituer autant de patrimoines littéraires nationaux, le Canada français

\footnotetext{
${ }^{1}$ Il écrit également : « Il y a bien sûr une histoire à faire des idées en NouvelleFrance - la forêt est là pour d'autres goûts, d'autres clairières - mais il est évident qu'en choisissant le début du régime colonial britannique je misais sur l'avènement du régime parlementaire et de l'imprimé pour retracer l'émergence d'une opinion publique.»(p.10) Bien que la Nouvelle-France occupe une place minime dans l'essai, les chapitres XII et XIII (p.383-432) éclairent les conditions d'émergence d'une littérature nationale au Canada français. Nova Doyon (2012) s'y intéresse de manière plus précise dans Formation des cultures nationales dans les Amériques, où elle compare le BasCanada au Brésil.
} 
semblait quant à lui procéder, dans la foulée de Camille Roy, au « travail d'élimination que l'historien impartial doit accomplir, et qu'il devra continuer ». De toute façon, ajoutait Roy, « [n]otre histoire de la littérature serait bien courte, s'il fallait n'y mettre encore que les disparus » (p. X). C'est ainsi que, jusqu'à la fin des années 1960 environ, l'histoire littéraire québécoise a le plus souvent privilégié les auteurs contemporains, autrement dit les vivants, au détriment des « disparus $»^{2}$.

Malgré ce mouvement de fond qui porte l'empreinte intellectuelle de $\mathrm{Roy}^{3}$, on remarque des changements dignes de mention dans les années 1970, notamment un regain d'intérêt pour le passé colonial du Canada français. Par exemple, dans le premier volume de l'Anthologie de la littérature québécoise (1978), consacré aux écrits de la Nouvelle-France, le préfacier Gilles Marcotte témoigne d'une réelle volonté de les mettre en valeur, tout en hésitant à leur reconnaître d'emblée un statut littéraire à part entière :

Toutes les sortes d'écriture sont ici mêlées: le sermon et le poème, l'histoire et la simple relation de voyage, la critique littéraire et le roman, le discours politique et le journal intime. Nous les réunissons dans le lieu commun de l'écriture, dans cet espace général du langage où tous les textes, si divers qu'ils soient, concourent à dessiner le visage d'une collectivité. N'estce pas cela, aussi, une littérature? (dans LeBlanc, p. XIII ; je souligne)

\footnotetext{
2 Par exemple, encore en 1967, Pierre de Grandpré, Léopold LeBlanc et Georges-Henri d'Auteuil écrivent en ouverture d'un chapitre portant sur les relations de voyage: "On n'a vu naître en Nouvelle-France aucune grande œuvre d'imagination ou de style. Ni les colons, ni les premiers Canadiens n'ont écrit de comédie ou de tragédie, de roman ou de recueil de poèmes. [...] Les premiers textes ne sont que des relations de voyage. Et ce genre continuera par-delà la fondation puis la cession du pays. » (p. 45)

3 Pour entrevoir l'influence considérable de Roy sur sa discipline dans la première moitié du 20e siècle, voir Cellard (p. 28-113).
} 
Une telle ouverture apparaît également, quoique de manière implicite, dans le premier tome du Dictionnaire des œuvres littéraires du Québec (1978), qui inclut notamment des entrées sur les œuvres de Champlain et de Sagard, de même qu'une sur les Relations des jésuites. D’ailleurs, une vingtaine d'années plus tard, le directeur du même Dictionnaire, Maurice Lemire, abordera de nouveau la question de la littérarité des textes du corpus colonial. Après avoir passé au crible certaines objections, y compris le fait qu'« [a]ucune histoire littéraire en France n'a retenu les noms de quelque relateur » (p. 16) ${ }^{4}$, il conclut que « ces textes peuvent être lus pour eux-mêmes indépendamment de leur valeur documentaire» (p. 19). Ainsi, «d'ores et déjà, on peut en considérer plusieurs comme de véritables textes littéraires » (ibid.). Les pages qui suivent montreront qu'à l'égard du patrimoine lettré de la Nouvelle-France, la tradition de l'histoire littéraire au Québec et au Canada ne s'est pas constituée sur des bases aussi inclusives. Au contraire, il semblerait que les textes du Régime français, quelle que fût leur nature, aient été victimes, pour plusieurs raisons et de différentes manières, de marginalisation.

\section{Réception des relations de voyage au Québec (1874-1957) : un survol}

Compte tenu des visées synthétiques du présent article, il m’apparaît nécessaire de préciser dans quel contexte

\footnotetext{
${ }^{4}$ Déjà, en 1954, Auguste Viatte émettait un jugement semblable : «Toute pratique qu'elle est, gardons-nous d'imaginer que cette littérature ait pour seul intérêt son contenu ». Puis, citant Gilbert Chinard : « En fait, voyageurs et missionnaires sont presque tous des humanistes, qui comme les Jésuites, ont "formé leur esprit à l'école de Virgile, de Tacite et surtout des Conciones" : leurs écrits fourmillent d'allusions aux Anciens. » (p. 26)
} 
interviennent les trois ouvrages retenus, chacun adoptant une perspective distincte, dans le discours sur les origines de la littérature québécoise et canadienne. Pour ce faire, je me référerai à un incontournable du 19e siècle, l'Histoire de la littérature canadienne d'Edmond Lareau, en plus de mettre en avant deux autres essais publiés respectivement en 1918 et 1957, le Manuel d'histoire de la littérature canadienne-française, de Camille Roy, ainsi que cet autre intitulé Littérature canadienne-française, de Samuel Baillargeon. Or, parmi les auteurs les plus souvent mentionnés dans ces ouvrages, figurent bon nombre de relateurs ${ }^{5}$ ou, du moins, de voyageurs.

Bien qu'il s'apparente parfois à l'inventaire et qu'il souffre de certains problèmes de structure, l'ouvrage de Lareau possède de nombreuses qualités. Pour le lecteur du $21^{\text {e }}$ siècle, son principal intérêt est de donner accès au point de vue d'un Canadien français cultivé de la seconde moitié du 19e siècle, non seulement sur le passé littéraire du Canada, mais aussi sur celui de toute l'Amérique coloniale. En effet, au lieu de se cantonner strictement à l'espace géographique occupé par le Québec de 1874, son panorama déborde ses frontières et mentionne pêlemêle Jean de Laet, Thomas Corneille, René de Laudonnière, Lescarbot, Champlain, Charles Lalemant, Lejeune, Sagard, Vincent Leblanc, Pierre Boucher, le père Marquette, Mgr de StVallier, Hennepin, Leclercq, Lahontan, les Lettres édifiantes et curieuses, Bacqueville de la Potherie, Lafitau, Charlevoix et Le Beau. Bien qu'il consacre peu de longs développements individuels à ces auteurs, certains passages révèlent assez

\footnotetext{
5 « Le mot relateur, utilisé par Labat dans sa préface de 1722 et par Littré, qui l'a lu chez Fénelon et Buffon, renverra tout naturellement à l'auteur [d'une relation de voyage]. » (Ouellet, p. 4)
} 
clairement ce qu'il pense des relations de voyage. Par exemple, au sujet de Champlain, il écrit: "C'est une des meilleures sources de notre histoire. Charlevoix dit que ses mémoires sont excellents pour le fond des choses et pour la manière simple et naturelle dont ils sont écrits. Il n'avance rien qu'il n'ait vu luimême ou sans avoir le témoignage de personnes sûres. » (Lareau, p. 39) De la même manière, il salue les qualités du témoignage de Nicolas Denys, auteur d'une Description géographique et historique des costes de l'Amérique septentrionale (1672) jugée très crédible :

L'auteur ne dit rien qu'il n'a vu de ses propres yeux. Dans le premier volume, il donne une description fort exacte du pays ; le second comprend l'histoire naturelle de la colonie et en particulier tout ce qui regarde la pêche de la morue. Il y étudie aussi les mœurs des sauvages et il termine en citant quelques traits historiques touchant les établissements de ceux qui partageaient avec lui la propriété et le gouvernement de l'Acadie et des environs. (p. 42)

Quant à Gabriel Sagard, dont la récente fortune éditoriale est enviable dans le corpus de la Nouvelle-France, il n'apprécie guère sa crédulité : «L'auteur raconte naïvement ce qu'il a vu en Canada où il demeura pendant quelque temps dans une mission huronne [...]. Son histoire n'a pas fourni beaucoup de matériaux utiles et importants aux écrivains qui l'ont suivi. » (ibid.)

Bref, ce qui importe à Lareau dans le genre viatique ${ }^{6}$, c'est d'abord et avant tout la fiabilité du témoignage des relateurs, du

\footnotetext{
${ }^{6}$ Bien sûr, la relation de voyage étant un genre polymorphe et composite, elle ne saurait se réduire à une forme convenue. Selon Motsch, il faudrait davantage la considérer comme une pratique discursive en constante évolution: "If the travel narrative in seventeenth-century France overtook the novel in the appreciation of the reader and imposed itself as the dominant epistemological model of the classical discourses of the period, [...] it remained nevertheless an intermediate genre. With the emergence of a more
} 
moment que cette qualité est soutenue par le style. Ainsi, au terme de son parcours pour le moins ambitieux et, du reste, sans précédent à l'époque, c'est sur un ton plutôt résigné qu'il conclut :

Tels sont, en résumé, les principaux écrivains français qui se sont occupés du Canada sous la domination française. Ces travaux n'ont plus à nos yeux l'importance qu'on leur accordait autrefois, malgré qu'ils soient toujours là pour décrire les antiquités américaines et les origines de la colonie. [...] De plus, les écrivains de la domination française ignorent, pour la plupart, la manière de classer les évènements, de les apprécier, de chercher les causes et d'analyser les époques. Ils se contentent de narrer simplement le fait dans un ordre méthodique et restreint, sans artifice dans l'arrangement des matières et sans pompe dans leur langage. [...] C'est pourquoi la lecture de ces mémoires est généralement ennuyeuse. (Lareau, p. 51-52)

Il faut souligner qu'au 19e siècle, une telle perplexité n'est pas isolée. En effet, on en trouve un écho dans l'Histoire de la littérature française hors de France, dont l'auteur, Virgile Rossel, plagie Lareau: «Humbles débuts assurément, que ceux de la littérature canadienne. Les premiers colons ne maniaient guère que la pioche. Il s'agissait pour eux de défricher et de labourer, non d'écrire. » (p. 308)7 Après un survol de trois pages, Rossel conclut en ces termes railleurs: "Quelques voyageurs, quelques annalistes, une sainte, voilà toute la littérature canadienne durant la domination française.» (p.311). Décidément, le $19^{\mathrm{e}}$ siècle n'aime pas les voyageurs ${ }^{8}$ !

realistic novel the travel narrative lost terrain, and did so not only in France. [...] To continue to compete for the favour of the audience, it would have to reorient and reinvent itself. » (p. 227).

${ }^{7}$ Cette idée à la mode sera plus tard contredite par Auguste Viatte : « Les pionniers de la Nouvelle-France ne manquent donc ni de culture ni du goût d'écrire. » (p. 2). Il le démontre d'ailleurs par la suite.

8 Cela est vraisemblablement lié au classement générique des œuvres à la fin du 19 e siècle, de même qu'à la conception des discours historiques, perçus 
Plus encore que Lareau, Camille Roy, à cause de son rayonnement au sein de l'institution littéraire canadiennefrançaise du début $20^{\mathrm{e}}$ siècle, a su imposer ses jugements esthétiques et idéologiques en participant activement à la construction d'une tradition littéraire nationale. S'il se révèle prolixe au sujet des auteurs du $19^{\mathrm{e}}$ siècle et de ses contemporains, insistant sur leur enracinement territorial et leurs thèmes de prédilection, il trouve fort peu à dire sur la Nouvelle-France. La raison de ce silence s'avère très simple: selon lui, l'histoire littéraire des Canadiens français ne commence pas avant le choc de $1760^{9}$. Bien entendu, précise-t-il,

[j]usqu'à cette époque, il y a bien eu, dans la Nouvelle-France, quelques manifestations de vie intellectuelle, des récits de voyage, comme ceux de Champlain, des relations fort intéressantes comme celles des Jésuites, des histoires comme celle de Charlevoix, des études de mœurs comme celles du Père Lafiteau [sic], des Lettres édifiantes et remplies de fines observations comme celles de Marie de l'Incarnation (Roy, p. 11-12).

Cela dit, puisque les ouvrages de cette époque furent « tous [sic] publiés en France » (p. 12) par des Français, Roy les exclut en bloc de l'histoire littéraire canadienne-française. Pourtant, lorsqu'il décrit la ville de Québec, qui a conservé après 1760 le statut de capitale intellectuelle acquis sous le Régime français, c'est à une relation de voyage flatteuse qu'il se réfère. Fait étonnant, l'auteur cité n'est pas un Français, mais bien un Finlandais de langue suédoise : « [Pehr] Kalm, qui visita la

comme au plus près du "degré zéro» de l'écriture, et ce, bien que les relateurs ne soient en aucun cas des historiens.

${ }^{9} \mathrm{Au}$ contraire, pour Viatte, dont la perspective continentale semble plus conforme au contexte de l'époque, "[l]a littérature française d'Amérique remonte aux débuts mêmes de la colonisation » (p. 1). 
Nouvelle-France en 1749 , et qui a laissé sur son voyage des notes si curieuses, et si sincères, a remarqué comme notre capitale réunissait alors les éléments d'une société distinguée, où le bon goût s'était conservé, où l'on se plaisait à le faire régner en ses manières, en son langage, en ses toilettes » (p. 1213). Les descriptions de Québec ne manquant pas chez les voyageurs français du $18^{\mathrm{e}}$ siècle, même les plus obscurs, on se serait attendu à lire des remarques plus connues, celles d'un Charlevoix par exemple (« Troisiéme Lettre», p. 234).

Enfin, malgré les sévères critiques essuyées au moment de sa publication ${ }^{10}$, la Littérature canadienne-française de Samuel Baillargeon se distingue des ouvrages de ses prédécesseurs par le ton employé et la présence marquée de l'iconographie. Il parvient également à concurrencer le Manuel de Roy «après cinquante ans de suprématie dans l'enseignement de la littérature nationale » (Cellard, p. 157). Il se révèle aussi plus inclusif que Roy à l'égard de la NouvelleFrance, considérant que ses «monuments écrits» ont suffisamment d'importance pour mériter une vingtaine de pages surprenantes, mais plutôt sympathiques, et ce, bien qu'ils « appartiennent à la littérature française » (Baillargeon, p. 23) :

Tous ces in-folio poudreux sont-ils à feuilleter pour saisir cette évolution sur le vif? Qu'il suffise de parcourir ceux dont la rédaction est plus soignée et le message plus substantiel. Ils nous communiquent une impression fraîche et nullement frelatée : le thème de la découverte. [...] Impressions naïves, remarques topographiques, coutumes ethnologiques, on trouve

\footnotetext{
10 Voir Cellard (p. 175-195). La plus sévère de ces critiques provient sans doute de l'historien Guy Frégault : « Reconnaissant avoir agi en tant que conseiller avec son collègue Maurice Séguin, Frégault précise que les deux professeurs de l'Université de Montréal ont prodigué à Baillargeon "le meilleur de tous les conseils qu'il a reçus : celui de ne pas publier son livre”. » (p. 180)
} 
de tout dans les écrits de ces explorateurs, appliqués à donner aux Français une idée du Canada. (ibid.)

À la suite de cette entrée en matière, il consacre en moyenne une page à Cartier, à Lescarbot, à Champlain, à Sagard et aux jésuites, tous des relateurs. Sur Champlain, figure incontournable qualifiée de «fondateur du Canada », l'auteur y va d'un jugement en demi-teinte : «Il a vécu assez longtemps au Canada pour s'adapter au pays; ses écrits en font foi. Quoique de médiocre valeur littéraire, ils révèlent un observateur minutieux, méticuleux même.» (p. 26) La constitution identitaire du Canadien occupant une large place dans la grille de lecture de Baillargeon, il ne faut pas s'étonner de la présence de critères comme l'adaptation et l'enracinement. Toutefois, en ce qui concerne la valeur littéraire, comme bien d'autres avant lui, il adopte une perspective impressionniste : Champlain écrit mal, voilà tout. De même, lorsque vient le temps d'aborder les Relations des jésuites, les plus célèbres voyageurs de la Nouvelle-France, il n'a d'autre choix que de se rallier à la tradition pour les encenser, à nouveau sans justifier clairement les raisons objectives de son dithyrambe : « Tous reconnaissent aux Relations une valeur documentaire indiscutable; on oublie parfois leur rare perfection littéraire, comparativement à la littérature du pays, à cette époque. L'authenticité pittoresque de ces pages héroïques leur donne une vraie valeur artistique. » (p. 28) Malgré les écarts et les erreurs factuelles qui émaillent l'ouvrage de Baillargeon, on ne saurait lui reprocher son manque d'enthousiasme à l'égard des textes qu'il a choisis pour représenter la Nouvelle-France.

Trois ouvrages récents : Canada anglais, Allemagne, Québec 
J'aimerais maintenant aborder trois ouvrages plus récents, qui me semblent remplir des fonctions pédagogiques similaires dans leur contexte respectif (canadien-anglais, germanophone et québécois), tout en s'adressant à des publics fort différents, sur le plan tant de la langue que des présupposés culturels. Aussi le choix du destinataire infléchit-il considérablement le traitement du sujet, comme c'était le cas dans les ouvrages abordés jusqu'ici, dont la trame reposait sur certains a priori récurrents. Évidemment, l'étudiant universitaire de langue allemande ne dispose pas du même bagage que son homologue canadien-anglais, et tous deux se distinguent du cégépien québécois contemporain. Il va sans dire que ces différences transparaissent dans la manière de présenter le corpus.

Tout d'abord, dans A History of Canadian Literature, William New consacre un chapitre à la production littéraire canadienne d'avant 1867, qu'elle soit écrite en français ou en anglais. Reflet d'une sensibilité contemporaine, un tel traitement de la réalité bilingue canadienne n'est pas nouveau en soi. En effet, dans Five-Part Invention: A History of Literary Histories in Canada, E. D. Blodgett constate que cette approche intégrée de l'histoire littéraire daterait des années 1920 au Canada anglais, sans toutefois être constante tout au long du 20 ${ }^{\mathrm{e}}$ siècle (p. 20-22). Ainsi, Archibald MacMechan et V. B. Rhodenizer affirment en chœur qu'il n'y a pas de production littéraire canadienne-française avant le 19e siècle, mais s'accordent aussi pour dire qu'il faut aborder la dualité constitutive du Canada : "In spite of their unlikeness, they [les anglophones et les francophones] have also a certain likeness; and any account of the beginnings of Canadian literature must 
reckon with these facts. » (p. 53) ${ }^{11}$ Quant à Lionel Stevenson, à la recherche d'un « homogeneous outlook », son " Manifesto for a National Literature» ne mentionne jamais la tradition francophone (p. 7 et 1-9).

Il va sans dire que, dans un contexte marqué par la Confédération et l'expansion vers l'ouest, les relations de voyage en Nouvelle-France n'occupent pas une place de choix. Ainsi, même quand Lorne Pierce les mentionne au passage, il ne tarde pas à les exclure du corpus canadien pour les raisons déjà invoquées par Roy :

From the appearance of Jacques Cartier's Voyages de découverte au Canada 1534-1552 [sic], to the close of the French régime, many priceless narrations of discovery, colonization and missionary entreprises were published in Paris and elsewhere. While these are our main sources of information covering this period, they do not belong to the body proper of Canadian literature. (p. 3)

L'ambition du bilinguisme mise à part, l'approche privilégiée par New diverge encore considérablement de celle de ses prédécesseurs, notamment en ce qui concerne le traitement réservé au corpus des relations de voyage au Canada datant de l'époque coloniale. En les traitant comme une masse de textes qui décrivent, à diverses époques et en plusieurs langues, le territoire qui forme désormais le Canada moderne, l'auteur extirpe de ces récits leur tradition respective, les décloisonne, ce qui rappelle leur existence simultanée dans un espace discursif donné. Autrement dit, James Cook aurait pu lire Charlevoix. Un autre trait d'importance soulevé par New concerne la valeur littéraire de ces relations, car si « [a]lmost all these explorers left observations and records [...]», souligne-t-

\footnotetext{
${ }^{11}$ Voir aussi Rhodeziner (p. 251-260).
} 
il, «[s]uch records vary markedly in literary quality » (p. 25). Les développements privilégiés dans le chapitre visent à étayer cette affirmation.

Après une mise en contexte historique soignée (p. 24-38), New consacre une vingtaine de pages à un substantiel corpus de relations de voyages, les regroupant en trois catégories: «Exploration journals » (p. 38-46), «Missionary journals » (p. 46-50) et « Travel, captivity and settlement journals » (p. 5056). Bien que les œuvres écrites en français restent minoritaires dans l'ensemble du chapitre, elles sont traitées sur le même plan que les autres, bien à leur place sur la ligne du temps. Ainsi, dans la première section, on relève deux petits paragraphes sur Cartier "who, like Adam in the garden, gave names to the land » (p. 39), un sur Champlain, dont l'essentiel porte sur la légende du Gougou ${ }^{12}$, et quelques phrases sur Hennepin, dont les œuvres sont qualifiées de « tangle of truths, half-truths, plagiarised adventures and wholesale fictions" (p. 40). En somme, selon New, « [t] he writers [des débuts de la Nouvelle-France] were torn [...] between devils and fables; between a belief in an active, effective spirit world that interfered in human behaviour, and a rationalist desire for more tangible explanations » (p.39). C'est un peu mince, compte tenu de la richesse du corpus, mais les synthèses de ce genre impliquent de douloureux sacrifices, surtout lorsqu'on cherche à rapprocher deux traditions qui, le plus souvent, semblent mener des vies parallèles ${ }^{13}$.

\footnotetext{
${ }^{12}$ Champlain en fait mention (p. 183-185).

13 New disposera d'un espace éditorial plus important dans son Encyclopedia of Literature in Canada (2002). On y trouve notamment des entrées sur Champlain, Sagard, les Relations des Jésuites et Lahontan.
} 
Contrairement à ce qu'on a observé dans la section précédente, l'essentiel du propos de «Missionary journals » concerne des relateurs francophones. Sans grande surprise, les Relations des jésuites tiennent le haut du pavé et quatre pages entières leur sont consacrées. Après avoir souligné l'influence marquante des Relations sur la tradition historiographique de langue anglaise, par le truchement de l'édition américaine de Reuben Gold Thwaites (1896-1901) et de l'œuvre de Francis Parkman, New en résume ainsi le contenu : "Read attentively, the Relations are indeed full of incident; they are also markedly varied in style, full of mundane details about crops and daily routine, and sometimes transparent in their exhaustion and frustration - with the work of the missions and with the political tensions of colonial life. » (p. 47) Plus loin, il souligne la difficile et nécessaire adaptation des missionnaires français à leur terre d'accueil parfois hostile et dénuée de tout ce qu'ils estimaient élémentaire. Autrement dit, «Canada was not France» (ibid.). En somme, l'auteur relève les principales caractéristiques des Relations, insistant sur la personnalité de certains relateurs (dont Brébeuf et Lejeune) et attirant l'attention sur les « human dimensions » (p. 48) qu'ils révèlent, sans pour autant négliger l'abondance des récits enchâssés et des variations stylistiques : "Some of the phrasing is casual and unornamented; some is clearly contrived, with balanced clauses and climactic arrangement.» (p.47) Au terme de ce développement, le lecteur a bien saisi que les Relations des jésuites sont riches et variées, ce qui leur assure une place à part dans la tradition viatique canadienne.

Quant à la dernière page de la section, elle s'avère beaucoup moins étoffée. Il est vrai que parmi les quatre religieuses mentionnées, Marie Morin, Marguerite Bourgeoys, 
Jeanne Mance et Marie de l'Incarnation, seules les trois dernières ont fait le voyage vers la Nouvelle-France (Marie Morin est née à Québec). De toute façon, puisque la vie religieuse semble de prime abord en parfaite contradiction avec la relation de voyage, New se concentre sur les aspects intimes de leur écriture.

Enfin, la troisième section consacrée aux voyageurs, «Travel, captivity and settlement journals», n'inclut aucun auteur francophone, à l'exception de St-John de Crèvecœur, en passant (p. 55). Or, ses Letters from an American Farmer (1782) furent rédigées en anglais, sa langue seconde, dans la toute jeune république américaine. Comme la Nouvelle-France ne manque pas d'aventuriers prompts à prendre la plume, il semble légitime de se demander où sont Radisson, Lahontan et Le Beau, qui maniaient tous aisément l'art du récit, dans le vrai comme dans le faux. Ces quelques réserves mises à part, le panorama offert par New se démarque, en raison notamment de sa perspective pancanadienne et de ses destinataires anglophones, de ceux qu'on trouve habituellement dans les ouvrages québécois du même type.

L'intérêt pour la littérature canadienne dans les deux langues officielles ne s'arrêtant pas aux frontières du pays, il est heureux que la recherche menée à l'étranger aboutisse elle aussi à des ouvrages de synthèse, indices d'une certaine dissémination dans le monde de l'enseignement universitaire. Ainsi, la rigoureuse Kanadische Literaturgeschichte [Histoire de la littérature canadienne] que nous allons maintenant examiner constitue «la première présentation générale de la littérature canadienne publiée en langue allemande » (Groß, Klooß et Nischik, p. VII ; je traduis). Malheureusement, pour des raisons 
linguistiques, les publications originales de ce genre circulent peu dans les milieux fréquentés par les spécialistes québécois et canadiens-anglais ${ }^{14}$. Aussi le public visé par la Kanadische Literaturgeschichte est-il germanophone (Allemagne, Autriche et Suisse) et universitaire (étudiants et professeurs).

Pourtant, l'intérêt de l'ouvrage ne tient pas qu'à sa langue de rédaction, mais bien davantage à sa structure, qui se distingue par un découpage périodique original, au corpus couvert et, surtout, à sa perspective extérieure qui réduit les interférences d'une idéologie nationale. C'est ce genre de renouvellement que permet un regard distant, où toutes les formes $d u$ «nous» se trouvent exclues. Ainsi, le premier chapitre ne traite pas de la production écrite des premiers voyageurs européens, mais bien de la tradition orale des Amérindiens («Die mündliche Literatur der Ureinwohner»), dont les origines sont millénaires. Quant au deuxième chapitre («Die Weißen kommen »), il esquisse un portrait général des explorations européennes entre l'an 1000 et 1600, donc de l'arrivée des voyageurs blancs. Outre Cabot, Verrazzano, Frobisher et Davis, l'auteur y mentionne Jacques Cartier et André Thevet. Bien que fort courts (douze pages en tout), ces deux chapitres donnent tout de suite le ton de l'ouvrage, en indiquant que rien ne sera négligé.

Cela dit, le troisième chapitre est le plus pertinent en ce qui concerne la Nouvelle-France. Il couvre les années 1604 à 1760 et les principaux auteurs y figurent, qui sont presque tous des relateurs: Champlain, Biard, Charles Lalemant, Sagard,

\footnotetext{
14 Par exemple, pour la plupart des chercheurs en littérature québécoise, l'ouvrage de Petr Kyloušek, Dějiny francouzsko-kanadské a quebecké literatury (2005), reste inaccessible sans traduction.
} 
Lejeune, Brébeuf, Boucher, Lescarbot, Hennepin, Lahontan, Lafitau, Charlevoix et même Radisson ${ }^{15}$. Au passage, KlausDieter Ertler souligne les procédés littéraires à l'œuvre dans le corpus viatique: "Puisque les relations de voyage et de colonisation consistent pour la plupart en une série de microrécits mâtinés de fiction, elles ne sont nullement dépourvues d'une dimension littéraire. ${ }^{16}$ »Plus loin, au sujet de Lejeune, il précise son jugement: «Malgré les contraintes rigoureuses imposées par l'ordre [des jésuites] quant à la fonction édifiante, dans les écrits de Lejeune, contrairement aux relations documentaires de Cartier et Champlain, les aspects littéraires ne sont pas relégués à l'arrière-plan. ${ }^{17}$ ». Enfin, son survol des Relations des jésuites l'amène à réfléchir à leur complexité générique: "Même par rapport au genre, il ne saurait y avoir de description univoque : même si, d'un point de vue fonctionnel, les lettres des jésuites se conforment au format de la relation annuelle prescrit par l'ordre, il n'en demeure pas moins qu'à l'intérieur de ce cadre se trouve une multiplicité de textes et de genres distincts. ${ }^{18} »$. En somme, Ertler esquisse en

15 Quant à la littérature coloniale de langue anglaise, elle est traitée par Konrad Groß et Dorothee Scholl au chapitre suivant, "Der Union Jack in Kanada (1763-1867) ». C'est donc dire que les auteurs ne tentent pas, contrairement à New, de fusionner les deux traditions.

16 «Da die Reise- und Kolonisierungsberichte meist eine Reihe von Mikroerzählungen mit fiktionalisierenden Einsprengseln darstellen, entbehren sie keineswegs einer literarischen Dimension. » (Ertler, 2005, p. 15 ; je traduis)

17 «Trotz der strengen Vorgaben des Ordens über die Erbauungsfunktion rückt in Lejeunes Texten im Gegensatz zu den dokumentarischen Berichten Cartiers oder Champlains die literarische Komponente nicht in den Hintergrund.» (ibid., p. 18 ; je traduis) Ces observations se trouvaient déjà dans l'introduction de Von Schwarzröcken und Hexenmeistern. Robes noires et sorciers. Jesuitenberichte aus Neu-Frankreich. Relations des Jésuites de la Nouvelle-France (1616-1649), dans Ertler (1997, p. 21).

18 "Auch hinsichtlich der Gattung lässt sich keine eindeutige Beschreibung geben : Wenngleich die Jesuitenbriefe in funktionaler Hinsicht dem Schema 
une vingtaine de pages un panorama plutôt équilibré de l'histoire et de la littérature de la Nouvelle-France, plus complet que dans la plupart des ouvrages québécois du même type publiés avant les années 1980 et, surtout, assez positif à l'égard des relations de voyage de toutes sortes. Après tout, elles constituent l'essentiel de son corpus publié à ce jour.

Enfin, pour revenir au Québec et à l'esprit de l'essai de Cellard, je dirai quelques mots sur une anthologie employée dans plusieurs cégeps, intitulée Confrontation des écrivains d'hier à aujourd'hui. Tome 3 : de la Nouvelle-France au Québec actuel. Soumis aux contraintes du manuel scolaire, dont les balises émanent du ministère de l'Éducation, l'ouvrage comporte six chapitres répartis sur 353 pages, le premier étant consacré aux «écrits de la Nouvelle-France ». Conformément à l'approche anthologique, les trente-sept pages contiennent à la fois des repères historiques, des images, des extraits à analyser ainsi que des questions. En plus de Lescarbot, longuement cité à la première page, deux chansons anonymes et six auteurs y figurent: Cartier, Champlain, Sagard, Marie de l'Incarnation, Élisabeth Bégon et Lahontan. Parmi ceux-ci, on relève donc pas moins de quatre relateurs. Quant aux deux femmes nommées, elles ont à tout le moins traversé l'Atlantique et raconté leur expérience du Nouveau Monde. Autrement dit, par le seul choix des textes présentés, les relations de voyage semblent perçues positivement par Raymond Paul, l'auteur du chapitre. D'ailleurs, les brefs paragraphes d'introduction le confirment, qui opposent l'« enthousiasme d'un Jacques Cartier qui s'émerveille de ce qu'il voit » à « la rationalité d'un observateur » (Paul, p. 5)

der vom Orden vorgeschriebenen Jahresbericht entsprechen, so können innerhalb dieses Rahmens eine Vielzahl von Textsorten und -gattungen festgestellt werden. » (Groß, Klooß et Nischik (dir.), p. 19 ; je traduis) 
comme Champlain. Plus loin, Paul écrit que le Grand voyage de Sagard « fournit une information de premier ordre sur un pays encore peu influencé par la présence européenne» (p.14), avant de présenter Lahontan comme la «victime d'une administration despotique » (p. 26). Le reste du chapitre est à l'avenant et, au final, cette anthologie initie un jeune public à la littérature de la Nouvelle-France, sans toutefois tomber dans les excès d'un Baillargeon.

Souvent considérées en bloc, sans classification autre que chronologique (avant 1763), soit pour être exclues de la tradition québécoise, soit pour y être intégrées, les œuvres constituant le corpus de la Nouvelle-France se révèlent donc difficiles à présenter dans les ouvrages d'histoire littéraire. En effet, dans un contexte discursif dominé par les généralités inhérentes à la synthèse, la réflexion sur les genres privilégiés dans le corpus colonial épouse des formes diverses, variant au gré de l'auteur qui s'empare du sujet ${ }^{19}$. Ainsi, contrairement à la plupart des ouvrages français du même type, où poésie romantique, roman réaliste et théâtre de boulevard profitent d'un traitement séparé, les écrits de la Nouvelle-France semblent le plus souvent ancrés dans une chronologie linéaire. Aussi le survol que nous venons de compléter nous a-t-il permis de constater, à tout le moins, qu'un questionnement théorique sur le genre n'est pas absolument nécessaire à l'inclusion d'œuvres dans une tradition littéraire, fussent-elles problématiques. Dans les faits, sans doute parce qu'elles représentent une large portion du corpus, les relations de voyage figurent dans toutes les histoires littéraires consultées,

19 Du reste, il existe des lieux nettement plus propices aux développements érudits. Voir Pioffet et Motsch (dir.) (2008), ainsi que Holtz et Motsch (dir.) (2011). 
et ce, même lorsque leurs auteurs ne consacrent qu'un développement minimal à la Nouvelle-France (voir Roy).

Ainsi, sur la base de l'échantillon analysé, certes limité mais néanmoins représentatif, nous pouvons affirmer que, toutes proportions gardées, les relations de voyage jouissent d'un statut appréciable par rapport aux autres œuvres du patrimoine lettré de la Nouvelle-France. Bien sûr, l'importance considérable des Relations des jésuites, de même que la circulation durable des œuvres de Champlain, Sagard et Lahontan, contribuent à cette visibilité. Enfin, notre modeste excursion hors des frontières actuelles du Québec, sur le plan tant territorial qu'intellectuel, a montré qu'un nouvel éclairage critique, libéré des ornières d'une longue tradition discursive, pouvait profiter aux œuvres du Régime français.

\section{Bibliographie}

BAILLARGEON, Samuel. (1957), Littérature canadienne-française, préface de Lionel Groulx, Montréal et Paris, Fides.

BlodgetT, Edward Dickinson. (2003), Five-Part Invention: A History of Literary Histories in Canada, Toronto, Buffalo et Londres, University of Toronto Press.

CEllaRd, Karine. (2011), Leçons de littérature. Un siècle de manuels scolaires au Québec, Montréal, Les Presses de l’Université de Montréal.

Champlain, Samuel de. (1993 [1603]), Des Sauvages, éd. Alain Beaulieu et Réal Ouellet, Montréal, Éditions Typo. 
CharlevoIX, François-Xavier. (1994 [1744]), «Troisième Lettre », dans Journal d'un voyage fait par ordre du roi dans l'Amérique septentrionale, vol.1, éd. Pierre Berthiaume, Montréal, Les Presses de l'Université de Montréal, coll. "Bibliothèque du Nouveau Monde », p. 211-236.

Doyon, Nova. (2012), Formation des cultures nationales dans les Amériques. Le rôle de la presse dans la constitution du littéraire au Bas-Canada et au Brésil au début du XIXe siècle, Québec, Les Presses de l'Université Laval.

ERTLER, Klaus-Dieter. (2005), «Fleur-de-Lys in Nordamerika (1604-1760) », dans Konrad GROß, Wolfgang KLOOß et Reingard M. NISCHIK (dir.), Kanadische Literaturgeschichte, Stuttgart et Weimar, Verlag J. B. Metzler, p. 12-30.

- (éd.). (1997), Von Schwarzröcken und Hexenmeistern. Robes noires et sorciers. Jesuitenberichte aus Neu-Frankreich. Relations des Jésuites de la Nouvelle-France (1616-1649), Berlin, Reimer.

GRANDPRÉ, Pierre de (dir.). (1967), Histoire de la littérature française du Québec, vol. 1, Montréal, Beauchemin.

GROß, Konrad, Wolfgang KLOOß et Reingard M. NISCHIK (dir.). (2005), Kanadische Literaturgeschichte, Stuttgart et Weimar, Verlag J. B. Metzler.

HolTz, Grégoire et Andreas Motsch (dir.). (2011), Éditer la Nouvelle-France, Québec, Les Presses de l'Université Laval.

KYLOUŠEK, Petr. (2005), Dějiny francouzsko-kanadské a quebecké literatury, Brno, Host.

LAMONDE, Yvan. (2000), Histoire sociale des idées au Québec (1760-1896), Montréal, Fides. 
LANSON, Gustave. (1965 [1910]), «La méthode de l'histoire littéraire ", dans Essais de méthode, de critique et d'histoire littéraire, éd. Henri PEYRE, Paris, Librairie Hachette, p. 31-56.

LAREAU, Edmond. (1874), Histoire de la littérature canadienne, Montréal, John Lovell.

LEBlanc, Léopold (éd.). (1978), Écrits de la Nouvelle-France, dans Gilles MARCOTTE (dir.), Anthologie de la littérature québécoise, vol. 1, Montréal, Les Éditions La Presse.

MaCMECHAN, Archibald. (1924), Head-Waters of Canadian Literature, Toronto, McClelland \& Stewart.

Motsch, Andreas. (2011), "Relations of Travel: Itinerary of a Practice ", Renaissance and Reformation / Renaissance et Réforme, vol. 34, no 1-2, Winter-Spring / hiver-printemps, p. 207-236.

NEW, William H. (ed.). (2002), Encyclopedia of Literature in Canada, Toronto, Buffalo et Londres, University of Toronto Press.

-. (1989), « Reporters: literature to $1867 »$, dans A History of Canadian Literature, Montréal \& Kingston, Londres \& Ithaca, McGill-Queen's University Press, p. 24-60.

OUELLET, Réal. (2010), La Relation de voyage en Amérique (XVIeXVIII e siècles). Au carrefour des genres, Québec, Les Presses de l'Université Laval.

PAuL, Raymond. (2007), "Écrits de la Nouvelle-France », dans André G. TuRcotTE (dir.), Anthologie. Confrontation des écrivains d'hier à aujourd'hui. Tome 3 : de la Nouvelle-France au Québec actuel, Montréal, Groupe Modulo, p. 1-37. 
PIERCE, Lorne. (1927), An Outline of Canadian Literature, Toronto, The Ryerson Press.

PIOFFET, Marie-Christine (dir) avec la collaboration d'Andreas MотSCH. (2008), Écrire des récits de voyage (XVe-XVIII siècles). Esquisse d'une poétique en gestation, Québec, Les Presses de l’Université Laval.

RHODEZINER, V. B. (1930), A Handbook of Canadian Literature, Ottawa, Graphic Publishers Limited.

RosSEL, Virgile. (1895), Histoire de la littérature française hors de France, Lausanne, Payot.

RoY, Camille. (1918), Manuel d'histoire de la littérature canadienne-française, Québec, L'Action sociale.

StEVEnSon, Lionel. (1926), Appraisals of Canadian Literature, Toronto, Macmillan.

Turcotte, André G. (dir.). (2007), Anthologie. Confrontation des écrivains d'hier à aujourd'hui. Tome 3 : de la Nouvelle-France au Québec actuel, Montréal, Groupe Modulo.

VAILlANT, Alain. (2010), L'Histoire littéraire, Paris, Armand Colin.

ViatTe, Auguste. (1954), Histoire littéraire de l'Amérique française des origines à 1950, Québec et Paris, Les Presses de l'Université Laval et PUF. 


\title{
Résumé
}

Dans cet article consacré à la réception institutionnelle du patrimoine lettré de la Nouvelle-France, je me concentre sur ce qu'on a écrit au sujet des relations de voyage dans quelques histoires de la littérature canadienne, canadienne-française et québécoise publiées depuis le XIX siècle. Peu goûtée par la critique historienne (souvent nationaliste), tant pour son origine exogène que pour sa nature informe et dénuée de prestige, la relation de voyage fait l'objet de commentaires mitigés. À l'aide d'un parcours chronologique et d'exemples contrastés, je dresserai un portrait général de la réception historiographique des œuvres appartenant à ce genre mal aimé.

\begin{abstract}
In this article addressing the institutional reception of the written heritage of New France, I am focussing on what has been said about travel writing in a selection of histories of Canadian, French-Canadian, and then Québécois literature, published since the $19^{\text {th }}$ century. Not truly appreciated by history-based criticism (often nationalist), as much for its exogenous origins as for its formless nature and lack of prestige, travel writing has generally been received with mixed feelings. By using a chronological approach and contrasted examples, I will sketch a general portrait of the historiographical reception of works pertaining to this unloved genre.
\end{abstract}

